\title{
Final Results of a 2:1 Control Case Observational Study with Interferon Beta and Interleukin-2 in Addition to First-Line Hormone Therapy in ER+ Endocrine Responsive Metastatic Breast Cancer Patients.
}

Andrea Nicolini ( $\square$ andrea.nicolini@med.unipi.it)

Universita degli Studi di Pisa https://orcid.org/0000-0002-5178-9510

\section{Giuseppe Rossi}

National Research Council: Consiglio Nazionale delle Ricerche

paola ferrari

Pisa University Hospital: Azienda Ospedaliero Universitaria Pisana

Riccardo Morganti

Pisa University Hospital: Azienda Ospedaliero Universitaria Pisana

Angelo Carpi

University of Pisa: Universita degli Studi di Pisa

\section{Research Article}

Keywords: breast cancer, metastasis, hormone-dependent, hormone resistance, immunotherapy

Posted Date: July 1st, 2021

DOl: https://doi.org/10.21203/rs.3.rs-533840/v1

License: (c) (i) This work is licensed under a Creative Commons Attribution 4.0 International License.

Read Full License 


\section{Abstract}

Following 22 additional months of post-operative follow-up and 6 further controls (total controls, $n=95$ ) here the final results of a 2:1 control-case retrospective observational study are shown. Controls were ER+HER-2- metastatic breast cancer patients who were given first-line hormone therapy $(\mathrm{HT})$ with aromatase inhibitors (Als) or fulvestrant. Moreover 28 of them (28.9\%) also received biological drugs including cyclin kinase inhibitors (CKis). Cases were $42 \mathrm{ER}+$ metastatic breast cancer patients who received the addition of interferon beta-interleukin-2 immunotherapy to first-line HT with selective estrogen receptor modulators/down-regulators (SERMs/SERDs). Median PFS and OS maintained significantly longer in the 42 studied patients who received hormone immunotherapy (HIT) than in the 95 controls (median time 33 vs 18 months, $p=0.002$ and 81 vs 62 months, $p=0.019$ ). In the analysis adjusted for disease-free interval (DFI), hormone receptor, HER-2 status, visceral involvement, Als and biological therapy, the PFS and OS hazard ratio (HR) further increased in favor of the 42 cases $(p=0.004$ and $p=$ 0.044 respectively). In the same type of patients treated with Als plus CKis a median PFS ranging from 25.3 to 28.18 months and a median OS of 37.5 months occurred. Overall, this strongly suggests multicentre randomised clinical trials to enter our proposed immunotherapy into clinical practice.

\section{Key Messages}

- In metastatic endocrine-dependent breast cancer patients acquired resistance is a common hurdle.

-The final results of a 2:1 control-case retrospective observational study are shown. ER+HER-2-controls received aromatase inhibitors (Als) or fulvestrant as first-line hormone therapy (HT) (some also received biological drugs including cyclin kinase inhibitors (CKi)). Cases were $\mathrm{ER}+$ patients treated with interferon beta-interleukin-2 immunotherapy plus selective estrogen receptor modulators/down-regulators.

-Median PFS and OS maintained significantly longer in the cases who received hormone immunotherapy than in the controls (median time 33 vs 18 months, $p=0.002$ and 81 vs 62 months, $p=0.019$ respectively). In the same type of patients treated with Als or fulvestrant plus CKi a median PFS ranging from 25.3 to 28.18 months and a median OS of 37.5 months occurred. Moreover, unlike CKis, our proposed immunotherapy did not show any relevant adverse event and the cost is about 8 to 18 times lower.

\section{Introduction}

ER+/luminal, including ER + HER2- breast cancer is the commonest type of metastatic breast cancer [13]. It is considered immunologically "cold" [4] therefore not suitable for immunological therapy. In this setting, the therapies interfering with E2 signaling, such as selective estrogen receptor modulators or downregulators (SERMs or SERDs) and aromatase inhibitors (Als), have been seminal in reducing breast cancer mortality over the past three decades [5]. Despite this, acquired resistance occurs in about $30 \%-50 \%$ ER + breast cancer patients on these hormonal therapies so requiring additional or substitutive therapy to maintain the clinical benefit [6-7]. Currently, first-line hormone therapy $(\mathrm{HT})$ with Als or 
fulvestrant is recommended in ER + HER-2- metastatic breast cancer patients [8]. Moreover, recently the CCND1-CDK4/6-RB pathway which is innately fundamental for the cell cycle control and governs whether a cell move on or arrests at the G1-S phase, has been recognized as a potential helpful molecular target to prolong the clinical benefit of ER + HER2 - luminal metastatic breast cancer on first-line hormonal therapy [9]. Therefore, some cyclin D-dependent kinase (CDK) 4/6 inhibitors (CKIs), particularly ribociclib [10], palbociclib [11] and lastly abemaciclib [12] following successful investigational clinical trials, have received FDA approval and are recommended in combination with Als or fulvestrant as first-line treatment of ER + HER2 - metastatic breast cancer patients. Contrary to the ongoing view, in 1992, we had hypothesized that the tumoral cell G0-G1 state due to the anti-proliferative action of the anti-estrogens in $\mathrm{ER}+$ metastatic breast cancer promoted a contemporaneous down-regulation of the mechanisms favoring the immune evasion [13]. According to our hypothesis, in these patients multiple ERs mediated mechanisms, including the immunological ones, rather than a single or few pathways accounted for the arising of the acquired resistance to conventional anti-estrogens. If so, during a clinical benefit on antiestrogens in the metastatic tumor microenvironment (TME) the tumoral G0-G1 cell state promoted counteraction/reversion of the multiple mechanisms sustaining tumor growth and the immune inhibition. This could allow interferon beta and interleukin-2 immunotherapy to stimulate an active immune response. First, in a pilot open-label study, patients receiving interferon beta and interleukin-2 in addition to conventional HT were compared with a small group of historical controls or with literature data where treated subjects were given conventional HT alone. This pilot study showed a more than doubled increase in progression-free (PFS) and overall (OS) survivals without any relevant side effect in patients also receiving immunotherapy [14]. Thereafter, due to the difficulties to launch a multicenter confirmatory randomized clinical trial we resorted to a 2:1 control case observational study where the studied patients in clinical benefit during first-line hormone therapy were compared with a relatively large group of comparable subjects who were not given additional immune therapy and were recruited at the same oncological department. In the first report [15] OS underwent a preliminary analysis and the Kaplan-Meyer curve was interrupted at 80 months due to the relatively short follow-up of the control group. Here, after more prolonged follow-up and some additional controls recruited, the final results are presented and discussed.

\section{Material And Methods}

\section{The study design and setting}

The study was a 2:1 ratio control-case observational study recruiting metastatic breast cancer patients in clinical benefit during first-line salvage HT. The enrolment interval was longer than usually because all patients were recruited with a relatively low recruitment rate at the same single oncological center. Also, it was at least in part different in cases (years 1992-2013) than in controls (years 2006-2018). The study started in 1992 as an open-label exploratory trial. Following the surprisingly promising and since 2005 more times reported results [14,16-20] we encountered unexpected difficulties in launching a sponsored prospective confirmatory randomised clinical trial likely because both the experimental drugs were at low cost with an expired license. On the other side bureaucracy revealed an insurmountable hurdle to launch 
a governmental trial. Then we decided to resort to a more feasible 2:1 control-case retrospective observational study. All data were collected from the charts of each recruited patient at the Oncology Department of Pisa University and were processed from April to October 2020. Following our initial report [15], 22 additional months of post-operative follow-up spent and 6 further controls were consecutively enrolled (total controls, $n=95$ ) then all data again have been analyzed and are briefly described here.

\section{Inclusion and exclusion criteria}

For both cases and controls inclusion criteria were: distant metastases stable or responsive to first-line SERMs, SERDs or Als in patients that had undergone primary mastectomy for breast cancer, an ECOG performance status $\leq 2$, white blood cells $\geq 3500 /$ microL, haemoglobin $\geq 10.5 \mathrm{~g} / \mathrm{dL}$, platelet count $>$ $125000 / \mathrm{microL}$, creatininemia $<1.5 \mathrm{mg} / \mathrm{dL}$, serum bilirubin $<1.5$ times the upper limito of normal, aspartate aminotransferase and alanine transferase $<3$ times the upper limito of normal, no severe and uncontrolled heart disease and availability to regularly carry out clinical-instrumental monitoring were also required. Previous or concomitant malignancy without a definite cure was an exclusion criterion both for cases and controls. The need of corticosteroids was an additional exclusion criterion for cases [1415].

\section{Cases}

All 42 recruited cases received first-line hormone immunotherapy (HIT) (Table 2)

Cases were enrolled according to the 2:1 ratio of the experimental design ( 2 controls for every patient studied) [15].

Conventional first-line HT and following therapeutic regimens

All 42 cases received SERMs as first-line salvage HT, i.e. tamoxifen ( 20 mg/day) (1992-1999 and 20032008) or toremifene (60 mg/day) (1998-2002), or Als, i.e. anastrozole (1 mg/day) or letrozole (2.5 $\mathrm{mg} /$ day) (2008-2013). At the progression, in 39 of the 42 enrolled patients, SERMs were replaced with Als. One of the 3 remaining progressing patients, who had been treated with Als as first-line salvage HT, was given fulvestrant, a more recent SERD and 1 received conventional chemotherapy (CT) due to antiestrogen resistance. The last patient is still responding to anastrozole. Patients progressing onto secondline salvage $\mathrm{HT}$ received the standard $\mathrm{CT}$, for most of them, cyclophosphamide methotrexate fluorouracil (CMF) and/or anthracyclines was the first regimen followed by vinorelbine and/or 5-FU as a successive regimen. Only a minority of the cases received a further $\mathrm{CT}$ regimen with a taxane alone or in combination.

Immunotherapy

After two months at least during which the metastatic disease of the candidates to recruitment had not progressed during conventional first-line salvage $\mathrm{HT}$ (induction time), all 42 recruited patients in addition to HT were given 3.000.000 IU of interferon beta i.m. every other day (3 times a week) for 4 weeks, 
followed by $3.000 .000 \mathrm{IU}$ of interleukin-2 s.c. every other day (3 times a week) for a further 4 weeks. For the successive 2 weeks, HT only was given to all the enrolled subjects then the same HIT schedule was started again. Thus, each cycle of HIT was 10 weeks long and HIT cycles were continued until progression. Four to 6 years after the beginning of the pilot study, the initial design of the study was adjusted. Interestingly, the rest interval between two successive cycles of immune therapy that lasted 4 weeks was decreased to 2 weeks and SERM daily dose, that during interferon beta treatment was increased, no more was changed [14]. All 42 patients gave written informed consent and the study was approved by the Council of the Department of Internal Medicine of Pisa University.

\section{Controls}

Controls were the first 95 consecutive metastatic breast cancer patients sharing the same eligibility criteria with the cases and treated from January 2006 to $10^{\text {th }}$ December 2018 at the same Oncology Centre, Department of Oncology, Pisa University Hospital.

Conventional first-line $\mathrm{HT}$ and following therapeutic regimens (Table 2).

Most controls were ER-positive HER2-negative patients who were given Als (letrozole, anastrozole or exemestane) or SERM/SERD (tamoxifen/fulvestrant) as first-line salvage HT. In most controls,_fulvestrant was the second-line salvage HT. Then standard chemotherapy was given to patients progressing onto second-line HT, for most of them, this was anthracyclines and/or taxanes and vinorelbine and/or 5-FU as first and second regimens, respectively.

Additional treatments to first-line salvage HT (Table 2).

Twenty-eight (28.9\%) controls in addition to first-line salvage HT received biological therapy. According to the ongoing guidelines the main aim of biological therapy (everolimus, bevacizumab, palbociclib) was to overcome or delay the occurrence of hormone resistance [21-22]. In 10 of these 28 controls receiving additional biological therapy, palbociclib, a CKi, was administered in combination with Als or SERMs/SERDs. Besides, 20 (21\%) peri/pre-menopausal controls were given LHRH agonists for up to 2 years, in $15(75 \%)$ of them, LHRH agonists were given in addition to SERM/SERD or Al and in the remaining 5 (25\%) LHRH agonists were administered with SERM/SERD or Al plus bevacizumab or palbociclib.

\section{Follow-up}

Disease-free interval (DFI) was the time from primary surgery for the metastatic disease ascertained by imaging techniques. On entry, a complete work-up to document the presence and the extent of metastatic disease was carried out in all recruited subjects. Bone scans, abdominal ultrasonography and chest X-ray together with the so-called 'gold standard' examinations (computed tomography (CT), magnetic resonance imaging (MRI) and positron emission tomography (PET)) were the instrumental tools used during the initial work-up. At the need, invasive cyto-histology procedure was additionally performed. CR, 
PR, SD and progressive disease (PD) were assessed according to the Response Evaluation Criteria in Solid Tumors (RECIST) 1.1 [23]. Every 2-4 months, all patients underwent control visits. Consistent with ASCO guidelines, routine blood examinations as, well as serum CEA-CA15.3 tumour marker (TM) panel measurement were regularly carried out at any control visit [24]. The conventional and/or gold standard instrumental examinations were commonly performed every 6-12 months by any patient recruited to the study for accurate monitoring of the metastatic disease. At the need, again, the cyto-histology procedure was used to ascertain or to confirm new lesions and metastatic disease progression. PFS was the interval time from the beginning of first-line salvage $\mathrm{HT}$ to metastatic disease progression documented by $\mathrm{CT}$ and/or MRI and/or PET, and cyto-histology when necessary. OS was the interval time from the beginning of first-line $\mathrm{HT}$ to the last observation or death for any reason. The last observation in the 137 patients took place on $30^{\text {st }}$ October 2020

\section{Statistical analysis}

Absolute and relative frequency were used to describe categorical data while mean and standard deviation described continuous data. Qualitative (gender, menopausal status, DFI, kind of response, hormone receptor status, Ki67/Mib-1 cut-off, HER2, site of metastases, number of lesions and Als as well as biological therapy) and quantitative (age and follow-up) variables according to therapy (HT or HIT), were compared by chi-square test and t-test (two-tailed) respectively. PFS and OS curves were built by the Kaplan-Meier method, and the log-rank test was applied to evaluate differences between curves. Cox regression models unadjusted and adjusted for DFI, hormone receptor and HER2 status, visceral involvement, Als and biological therapy were also used. The Ki67/Mib-1 rate has not been included in the adjusted analysis due to the high number of missing data. The results of the Cox regression were expressed using both the hazard ratio (HR) with its related $95 \%$ confidence interval $(95 \% \mathrm{Cl})$ and regression coefficients (RC). Differences were considered significant at $p<0.05$. All analyses were carried out using SPSS v. 27 technology. PFS was the primary endpoint, and the Kaplan-Meier curve has been described up to the last observation.

\section{Results}

There was a statistically significant difference in the mean follow-up time $(p<0.0001)$, HER2 positive $(p<0.0001)$, visceral metastases $(p=0.003)$ and the rate of patients with Ki67/Mib-1 $>25 \%(p<0.0001)$ in favour of the cases. Conversely, the percentage of patients with a DFI $\leq 24$ months $(p=0.008)$ and ERpositive patients $(p<0.0001)$, was significantly higher in the controls (Table 1$)$. Table 2 also shows that a significantly higher proportion of the controls than the cases were given Als and biological therapy. In this updating report compared with the previous one [15] PFS and OS maintained significantly longer in the 42 studied patients than in the 95 controls $(p=0.002$ and $p=0.019$ respectively in the unadjusted analysis) (Figure 1 A-B). Particularly the median time was 33 vs 18 months for PFS and 81 vs 62 months for OS in the 42 studied patients compared to the 95 controls. In the unadjusted analysis the PFS HR was 1.902 and that of OS, it was 1.684 in favor of the 42 studied patients. In the adjusted analysis, the PFS HR further increased to 2.533 and that of OS to 2.158 in favor of the 42 studied patients (Table 3). 
Cumulative survival at 10 years was $15 \%$ in the 42 studied patients and $7 \%$ in the 95 comparable controls. One of the 42 studied patients with oligometastatic bony disease [25-27] is in CR more than 12 years from the beginning of first-line hormone therapy [28]. Different tissue immune patterns and tumor microenvironments [29-30] have been reported as well as that bone metastases are immune preserved [30-31]. Despite this, during first line HIT, no significant discrepancy of metastatic disease evolution occurred in different tissues. In fact, in the 14 cases with an initial metastatic involvement of more organs (see Table 1), the same evolution (CR, PR, SD during clinical benefit and PD at the progression) was contemporaneously observed by the instrumental examinations in the 2 (12 cases) or 3 ( 2 cases) involved organs.

\section{HIT tolerability}

Good HIT tolerability was confirmed [14]. No grade 3-4 adverse event was reported. Besides grade 0-1 or grade 1-2 flu-like syndrome and injection site reaction were the commonest side effects which occurred in about $80 \%$ and $90 \%$ of the cases respectively.

\section{Discussion}

ER positive luminal breast cancer is considered an immunologically "cold" breast cancer subtype

In breast cancer, anti-HER-2 monoclonal antibodies and PD-L1 inhibitors, combined with conventional chemotherapy, are currently the only immunotherapy used in clinical practice. The former is given in HER-

2 positive [32] and the latter in triple negative breast cancer patients (TNBC) [33]. However, ER positive, including ER+ HER-2-, luminal breast cancer represents $60 \%$ to $80 \%$ of all breast malignancies and the incidence increases with older age [3,34]. ER positive luminal breast cancer is considered immunologically "cold" [4] therefore not suitable for immunological therapy. Unlike this, the addition of interferon beta-interleukin-2 immunotherapy to first-line salvage HT prolonged PFS and OS in an initial open-label exploratory clinical trial compared to 30 historical controls and literature data [14]. Despite these surprisingly promising and since 2005 more times reported results [13-14,16-20], we failed in launching a sponsored randomized confirmatory trial. Therefore, we resorted to a more feasible 2:1 control-case retrospective observational study conducted in a single oncologic centre [15].

\section{Main different characteristics of cases compared with controls: impact on PFS and OS}

The different recruitment interval time was the reason for most discrepancies. By far it is known that ER and/or PgR positive breast cancer patients are expected to respond to anti-estrogen therapy. However, roughly $20 \%$ of false negative rate of hormone receptor status evaluation by $\mathrm{IHC}$ for different reasons had been reported [35-36]. So, mainly in the first half (years 1992-2003) of the interval time of cases recruitment, those in clinical benefit during first-line anti-estrogen salvage therapy (induction time) were enrolled even if they were ER negative. Therefore 10 (23.8\%) of the 42 cases were ER-negative $(p<0.0001)$ including three triple-negative breast cancer (TNBC) patients. For the same reason, 10 other cases were HER-2 positive (Table 1). At the end of nineties, following molecular subtype classification, ER positive 
HER-2 negative breast cancer patients were the selected population recommended by ongoing guidelines [21-22] to receive first-line anti-estrogen treatment. The interval time of controls' recruitment (years 20062018) followed that of cases (years 1992-2013). This accounts for more controls than cases being ER positive (100\%) HER-2 negative (95.8\%). The difference in accrual period again accounted for a higher proportion of controls treated with Als ( $82 \%$ vs $7.1 \%$ ) while SERMs/SERDs were given to $92.9 \%$ of the cases and $18 \%$ only of the control group $(p<0.0001)$. Also, over the time SERMs (tamoxifen or toremifene) were replaced by Als due to a clear superiority of Als versus SERMs in adjuvant and metastatic settings [37-38]. Moreover 28 (28.9\%) of the 95 controls were given molecular-targeted drugs (everolimus, palbociclib, bevacizumab) to overcome hormone resistance and prolong the clinical benefit during first line hormone therapy [21-22]. Instead, no case received any of these drugs which were not available or recommended at the time of their metastatic disease. ER-positive/HER2-negative patients take part in the luminal molecular subtype, namely the type with better prognosis [39-40] while TNBC is that with the worst prognosis [39-40]. Accordingly, ER negative/ HER-2 positive compared with ER positive/HER-2 negative are widely recognized unfavorable prognostic/predictive markers [41-42]. Moreover, as to the 10 HER-2 positive cases, 8 of them could not receive anti-HER-2 specific therapy due to the lack of availability of any of these types of drugs at the time of their metastatic disease. The two remaining patients among the different lines successive to hormone therapy were given lapatinib concomitant with capecitabine that was interrupted after 3-4 months following heavy side effects (diarrhea). Most cases $(92.9 \%)$ received SERMs/SERDs, unlike most controls that received Als (82\%) as first-line HT. However, while the mean clinical benefit of first-line tamoxifen has been reported to be about 13 months, that of Als was 3 months at least longer [37-38]. In addition, cases, unlike some controls could not take benefit from molecular target therapies which again were not available at the time of their metastatic disease. Also, visceral metastases more often occurred in cases than in controls. Overall, the principal prognostic/predictive characteristics (hormone receptor and HER 2 status, Als as well as biological therapy and visceral involvement) except DFI of the patients recruited into the cases and controls were significantly in favour of controls (Table 1). Therefore, these discrepancies were expected to prolong median PFS and OS in the 95 controls compared with 42 cases. When all these factors were taken into consideration in the adjusted statistical analysis, both the end-points maintained a significant difference in favor of the 42 cases. Moreover, the HR for PFS and OS that was 1.902 and 1.684 in the unadjusted analysis increased to 2.533 and 2.158 respectively in the adjusted one in favour of cases (Table 3 ). Lastly, most of the 42 cases unlike the 95 controls could not benefit from the introduction of taxanes into current clinical practice [21-22]. This likely accounts for lower significance of OS $(p=0.019)$ than PFS $(p=0.002)$ in the cases compared with controls.

\section{CKis in addition to Als or fulvestrant}

In metastatic ER+ HER2 negative breast cancer patients, CKis in addition to Als or fulvestrant, are currently recommended. These drugs which mainly are addressed to inhibit the G1-S checkpoint have been recently investigated. Early randomized clinical trials conducted with these drugs, in particular, ribociclib, palbociclib and more recently with abemaciclib have shown significant prolongation of PFS when added to Als or fulvestrant. Therefore, these drugs received prompt FDA approval to enter into 
clinical practice. In clinical trials carried out with CKis in addition to Als or fulvestrant, median PFS ranged from 25.3 with ribociclib [10] to 28.2 months with abemaciclib [12]. In ribociclib and abemaciclib trials, median OS has not yet been reached, however mature data in palbociclib trial [11], did not show any significant difference in treated patients vs controls (34.5 vs 37.5 months). Grade 3-4 AEs from any cause have been reported in $>10 \%,>15 \%$ and in $58 \%$ of patients in the arm additionally treated with ribociclib, palbociclib and abemaciclib respectively. Particularly grade 3 neutropenia occurred in $>50 \%$ of patients additionally receiving ribociclib or palbociclib and in $22 \%$ with abemaciclib. In our observational 2:1 control-case study, PFS in the 42 cases treated with SERMs/SERDs plus immunotherapy was longer (31 months) than in clinical trials using CKis in addition to Als or fulvestrant (from 25.3 to 28.2 months). However, in our 42 cases no 3-4 grade AEs occurred and grade 1-2 flu-like syndrome (50\%) and injection site reaction (61\%) were the only more serious commonly observed AEs [14]. Furthermore, our proposed immune-therapy is 8 to 18 times cheaper than the CKis.

\section{Potential mechanistic rationale of HIT}

The association of our immunotherapy to anti-estrogens in ER+ metastatic breast cancer patients was based on the hypothesis that anti-estrogens reversed the inhibition of the immune system in the TME thus allowing an immune stimulation of the effector T cells by interferon beta interleukin-2 sequence. Recently the potential of anti-estrogens to revert the immunosuppressive TME has been highlighted [43]. The G0-G1 state induced by anti-estrogens likely favour the stimulation of the effector immune cells. In our initial open-label exploratory clinical study also immunologic laboratory data supported this effect $[17,44]$. The immune stimulation by beta-interferon and interleukin-2 uses a physiological pathway. This may explain why no important AEs occurred. Differently, the inhibition of the G1-S check point by the CKis involving tumoral and non-tumoral cells may account for the occurrence of some relevant AEs reported in the above-mentioned clinical trials. Despite the limitation of our study, which is retrospective and observational, the persistence of the promising results over a long time confirms our rationale and suggests that an active immune stimulation in metastatic patients in clinical benefit during first-line salvage hormone therapy is the main road to investigate. Interestingly our proposed immunotherapy may be added to Als CKis combination which implements an anti-proliferative action.

\section{Conclusions}

Overall, these findings strongly suggest multi-centre randomised confirmatory clinical trials to eventually enter our proposed immunotherapy into clinical practice.

\section{Declarations}

\section{Funding}

No funding. 
No conflict of interest.

\section{Ethics approval and consent}

All patients gave witnessed written informed consent and the study was approved by the Council of the Department of Internal Medicine of Pisa University. The study was performed in accordance with the Declaration of Helsinki.

\section{Availability of data and material}

All data used are available in the archive of the Department of Oncology, Oncologic Centre of Pisa University Hospital.

\section{Author contributions}

All authors had full access to all data of the submitted study.A. N. conceived the initial experimental design, conducted the study and wrote the manuscript, G. R. conceived the retrospective control-case observational study and carried out statistical analysis of previously published reports, P. F. conducted the study and revised the manuscript, R. M. carried out statistical analysis, A. C. conducted the study and revised the manuscript.

\section{References}

1. Howlader N, Altekruse SF, Li Cl, Chen VW, Clarke CA, Ries LA, Cronin KA (2014) US incidence of breast cancer subtypes defined by joint hormone receptor and HER2 status. J Natl Cancer Inst 106(5), dju055.

2. Parise CA, Bauer KR, Brown MM, Caggiano V (2009) Breast cancer subtypes as defined by the estrogen receptor (ER), progesterone receptor (PR), and the human epidermal growth factor receptor 2 (HER2) among women with invasive breast cancer in California, 1999-2004. Breast J 15(6), 593602.

3. Senkus E, Cardoso F, Pagani O (2014) Time for more optimism in metastatic breast cancer? Cancer Treat Rev 40(2), 220-228.

4. Vonderheide RH, Domchek SM Clark AS (2017) Immunotherapy for Breast Cancer: What Are We Missing? Clin Cancer Res 23(11), 2640-2646.

5. Caswell-Jin JL, Plevritis SK, Tian L, Cadham CJ, Xu C, Stout NK, Sledge GW, Mandelblatt JS, Kurian AW (2018) Change in Survival in Metastatic Breast Cancer with Treatment Advances: Meta-Analysis and Systematic Review. JNCl Cancer Spectr 2(4), pky062.

6. Nayar U, Cohen O, Kapstad C, Cuoco MS, Waks AG, Wander SA, Painter C, Freeman S, Persky NS, Marini L, Helvie K, Oliver N, Rozenblatt-Rosen O, Ma CX, Regev A, Winer EP, Lin NU, Wagle N. 
(2019)Acquired HER2 mutations in ER+ metastatic breast cancer confer resistance to estrogen receptor-directed therapies. Nat Genet 51(2), 207-216.

7. AlFakeeh A, Brezden-Masley C (2018) Overcoming endocrine resistance in hormone receptor-positive breast cancer. Curr Oncol 25(Suppl 1), S18-S27.

8. Najim O, Seghers S, Sergoynne L, Van Gaver H, Papadimitriou K, Wouters K, Trinh XB, Huizing MT, Tjalma W (2019) The association between type of endocrine therapy and development of estrogen receptor-1 mutation(s) in patients with hormone-sensitive advanced breast cancer: A systematic review and meta-analysis of randomized and non-randomized trials. Biochim Biophys Acta Rev Cancer 1872(2), 188315.

9. Piezzo M, Cocco S, Caputo R, Cianniello D, Gioia GD, Lauro VD, Fusco G, Martinelli C, Nuzzo F, Pensabene M, Laurentiis M (2020) Targeting Cell Cycle in Breast Cancer: CDK4/6 Inhibitors. Int J Mol Sci 21(18), 6479.

10. Hortobagyi GN, Stemmer SM, Burris HA, Yap YS, Sonke GS, Paluch-Shimon S, Campone M, Petrakova K, Blackwell KL, Winer EP, Janni W, Verma S, Conte P, Arteaga CL, Cameron DA, Mondal S, Su F, Miller M, Elmeliegy M, Germa C, O'Shaughnessy J (2018) Updated results from MONALEESA-2, a phase III trial of first-line ribociclib plus letrozole versus placebo plus letrozole in hormone receptor-positive, HER2-negative advanced breast cancer. Ann Oncol 29(7), 1541-1547.

11. Finn RS, Boer, K Bondarenko I, Patel R, Pinter T, Schmidt M, Shparyk YV, Thummala A, Voitko N, Bananis E, McRoy L, Wilner K, Huang X, Kim S, Slamon DJ, Ettl J (2020) Overall survival results from the randomized phase 2 study of palbociclib in combination with letrozole versus letrozole alone for first-line treatment of ER+/HER2- advanced breast cancer (PALOMA-1, TRIO-18). Breast Cancer Res Treat 183(2), 419-428.

12. Johnston S, Martin M, Di Leo A, Im SA, Awada A, Forrester T, Frenzel M, Hardebeck MC, Cox J, Barriga S, Toi M, Iwata H, Goetz MP (2019) MONARCH 3 final PFS: a randomized study of abemaciclib as initial therapy for advanced breast cancer. NPJ Breast Cancer 5, 5 .

13. Nicolini A, Carpi A, Ferrari P, Biava PM, Rossi G (2016) Immunotherapy and Hormone-therapy in Metastatic Breast Cancer: A Review and an Update. Curr Drug Targets 17(10), 1127-1139.

14. Nicolini A, Carpi A (2005) Beta-interferon and interleukin-2 prolong more than three times the survival of 26 consecutive endocrine dependent breast cancer patients with distant metastases: an exploratory trial. Biomed Pharmacother 59(5), 253-263.

15. Nicolini A, Rossi G, Ferrari P, Morganti R, Carpi A (2020) A new immunotherapy schedule in addition to first-line hormone therapy for metastatic breast cancer patients in a state of clinical benefit during hormone therapy. J Mol Med (Berl) 98(3), 375-382.

16. Nicolini A, Carpi A, Rossi G (2005) An immunotherapy schedule in endocrine-dependent metastatic breast cancer: correlation between clinical course and immunologic parameters. J Immunother 28(3), 276-279.

17. Nicolini A, Carpi A, Rossi G (2007) Relationship of cellular immunity, cytokines and CRP with clinical course in breast cancer patients with endocrine-dependent distant metastases treated with 
immunotherapy. Cancer Lett 251(2), :330-338.

18. Nicolini A, Carpi A, Ferrari P, Rossi G (2008) Immunotherapy prolongs the serum CEA-TPA-CA15.3 lead time at the metastatic progression in endocrine-dependent breast cancer patients: a retrospective longitudinal study. Cancer Lett 263(1), 122-129.

19. Nicolini A, Ferrari P, Kotlarova L, Rossi G, Biava PM (2015) The PI3K-AKt-mTOR Pathway and New Tools to Prevent Acquired Hormone Resistance in Breast Cancer. Curr Pharm Biotechnol 16(9), 804815.

20. Nicolini A, Barak V, Biava PM, Ferrari P, Rossi G, Carpi A (2019) The Use of Immunotherapy to Treat Metastatic Breast Cancer. Curr Med Chem 26(6), 941-962.

21. https://www.nccn.org/professionals/physician_gls/pdf/breast.pdf. Accessed February 20, 2021

22. https://www.esmo.org/Guidelines/Breast-Cancer. Accessed February 20, 2021

23. Eisenhauer EA, Therasse P, Bogaerts J, Schwartz LH, Sargent D, Ford R, Dancey J, Arbuck S, Gwyther S, Mooney M, Rubinstein L, Shankar L, Dodd L, Kaplan R, Lacombe D, Verweij J (2009) New response evaluation criteria in solid tumours: revised RECIST guideline (version 1.1). Eur J Cancer 45(2), 228247.

24. Bast RC Jr, Ravdin P, Hayes DF, Bates S, Fritsche H Jr, Jessup JM, Kemeny N, Locker GY, Mennel RG, Somerfield MR, American Society of Clinical Oncology Tumor Markers Expert Panel (2001) 2000 update of recommendations for the use of tumor markers in breast and colorectal cancer: clinical practice guidelines of the American Society of Clinical Oncology. J Clin Oncol 19(6), 1865-1878.

25. David S, Tan J, Savas P, Bressel M, Kelly D, Foroudi F, Loi S, Siva S (2020) Stereotactic ablative body radiotherapy (SABR) for bone only oligometastatic breast cancer: A prospective clinical trial. Breast 49, 55-62.

26. Coleman RE, Smith P, Rubens RD (1998) Clinical course and prognostic factors following bone recurrence from breast cancer. Br J Cancer 77(2), 336-340.

27. Pagani O, Senkus E, Wood W, Colleoni M, Cufer T, Kyriakides S, Costa A, Winer EP, Cardoso F, ESOMBC Task Force (2010) International guidelines for management of metastatic breast cancer: can metastatic breast cancer be cured? J Natl Cancer Inst 102(7), 456-463.

28. Nicolini A, Ferrari P, Morganti R, Carpi A (2019) Treatment of Metastatic or High-Risk Solid Cancer Patients by Targeting the Immune System and/or Tumor Burden: Six Cases Reports. Int J Mol Sci 20(23), 5986.

29. Oliver AJ, Lau PKH, Unsworth AS, Loi S, Darcy PK, Kershaw MH, Slaney CY (2018) Tissue-Dependent Tumor Microenvironments and Their Impact on Immunotherapy Responses. Front Immunol 9, 70.

30. Lee H, Na KJ, Choi H (2021) Differences in Tumor Immune Microenvironment in Metastatic Sites of Breast Cancer. Front Oncol 11, 649004.

31. Roato I, Ferracini R (2018) Cancer Stem Cells, Bone and Tumor Microenvironment: Key Players in Bone Metastases. Cancers (Basel) 10(2), 56. 
32. Escrivá-de-Romaní S, Arumí M, Bellet M, Saura C (2018) HER2-positive breast cancer: Current and new therapeutic strategies. Breast 39, 80-88.

33. Kwa MJ, Adams S (2018) Checkpoint inhibitors in triple-negative breast cancer (TNBC): Where to go from here. Cancer 124(10), 2086-2103.

34. Howlader N, Altekruse SF, Li Cl, Chen VW, Clarke CA, Ries LA, Cronin KA (2014) US incidence of breast cancer subtypes defined by joint hormone receptor and HER2 status. J Natl Cancer Inst 106(5), dju055.

35. Alberts SR, Ingle JN, Roche PR, Cha SS, Wold LE, Farr GH Jr, Krook JE, Wieand HS (1996) Comparison of estrogen receptor determinations by a biochemical ligand-binding assay and immunohistochemical staining with monoclonal antibody ER1D5 in females with lymph node positive breast carcinoma entered on two prospective clinical trials. Cancer 1978(4), 764-772.

36. Allred DC, Bustamante MA, Daniel CO, Gaskill HV, Cruz AB (1990) Jr. Immunocytochemical analysis of estrogen receptors in human breast carcinomas. Evaluation of 130 cases and review of the literature regarding concordance with biochemical assay and clinical relevance. Arch Surg, 125(1), 107-13.

37. Mouridsen H, Gershanovich M, Sun Y, Perez-Carrion R, Boni C, Monnier A, Apffelstaedt J, Smith R, Sleeboom HP, Jaenicke F, Pluzanska A, Dank M, Becquart D, Bapsy PP, Salminen E, Snyder R, ChaudriRoss H, Lang R, Wyld P, Bhatnagar A (2003) Phase III study of letrozole versus tamoxifen as first-line therapy of advanced breast cancer in postmenopausal women: analysis of survival and update of efficacy from the International Letrozole Breast Cancer Group. J Clin Oncol 21(11), 2101-2109.

38. Thürlimann B, Hess D, Köberle D, Senn I, Ballabeni P, Pagani O, Perey L, Aebi S, Rochlitz C, Goldhirsch A (2004) Anastrozole ('Arimidex') versus tamoxifen as first-line therapy in postmenopausal women with advanced breast cancer: results of the double-blind cross-over SAKK trial 21/95-a sub-study of the TARGET (Tamoxifen or 'Arimidex' Randomized Group Efficacy and Tolerability) trial. Breast Cancer Res Treat 85(3), 247-254.

39. Kim YJ, Kim JS, Kim IA (2018) Molecular subtype predicts incidence and prognosis of brain metastasis from breast cancer in SEER database. J Cancer Res Clin Oncol 144(9), 1803-1816.

40. Kalimutho M, Parsons K, Mittal D, López JA, Srihari S, Khanna KK (2015) Targeted Therapies for Triple-Negative Breast Cancer: Combating a Stubborn Disease. Trends Pharmacol Sci 36(12), 822846.

41. Pusina S (2018) Correlation of Serum Levels of Urokinase Activation Plasminogen (uPA) and Its Inhibitor (PAl-1) with Hormonal and HER-2 Status in the Early Invasive Breast Cancer. Med Arch 72(5), 335-340.

42. Nicolini A, Ferrari P, Duffy MJ (2018) Prognostic and predictive biomarkers in breast cancer: Past, present and future. Semin Cancer Biol 52, 56-73.

43. Welte T, Zhang XH, Rosen JM (2017) Repurposing Antiestrogens for Tumor Immunotherapy. Cancer Discov 7(1), 17-19. 
44. Nicolini A, Rossi G, Ferrari P, Carpi A (2014) Clinical and laboratory patterns during immune stimulation in hormone responsive metastatic breast cancer. Biomed Pharmacother 68(2), 171-178.

\section{Tables}

Table 1. Principal characteristics of the 137 metastatic breast cancer patients in clinical benefit during hormone therapy. 


\begin{tabular}{|c|c|c|c|}
\hline Patients characteristic & $\begin{array}{l}\text { Controls } \\
\text { (1st line HT) }\end{array}$ & $\begin{array}{l}\text { Cases } \\
\text { (1st line HIT) }\end{array}$ & p-value \\
\hline$N$ & 95 & 42 & \\
\hline Gender & & & 0.919 \\
\hline Female & $93(97.9 \%)$ & $41(97.6 \%)$ & \\
\hline Male & $2(2.1 \%)$ & $1(2.4 \%)$ & \\
\hline Menopausal status & & & 0.788 \\
\hline Post-menopausal & 75 (78.9\%) & $34(80.9 \%)$ & \\
\hline Pre-perimenopausal & $20(21.1 \%)$ & $8(19.1 \%)$ & \\
\hline Age (yrs), average, range & $61.4(36-89)$ & $60.1(34-82)$ & 0.577 \\
\hline Follow-up (mo), mean \pm sd, range & $52.6 \pm 24.7(14-149)$ & $86.2 \pm 41.3(31-221)$ & $<0.0001$ \\
\hline$D F I$ & & & 0.008 \\
\hline$>24 \mathrm{mo}$ & $60(63.2 \%)$ & $36(85.7 \%)$ & \\
\hline$\leq 24 \mathrm{mo}$ & $35(36.8 \%)$ & $6(14.3 \%)$ & \\
\hline Kind of response & & & 0.051 \\
\hline CR & $1(1 \%)$ & $4(9.6 \%)$ & \\
\hline PR & $33(34.8 \%)$ & $13(30.9 \%)$ & \\
\hline SD & $61(64.2 \%)$ & $25(59.5 \%)$ & \\
\hline Hormone receptor status & & & $<0.0001^{1}$ \\
\hline $\mathrm{ER}+/ \mathrm{PR}+$ & $84(88.4 \%)$ & $21(50 \%)$ & \\
\hline $\mathrm{ER}+/ \mathrm{PR}-$ & $11(11.6 \%)$ & $6(14.3 \%)$ & \\
\hline ER-/PR+ & 0 & $1(2.4 \%)$ & \\
\hline ER-/PR- & 0 & $9(21.4 \%)$ & \\
\hline NA & 0 & $5(11.9 \%)$ & \\
\hline Ki67/Mib-1 cut-off [34] & & & $<0.0001$ \\
\hline$>25 \%$ & $20(21 \%)$ & $13(30.9 \%)$ & \\
\hline$\leq 25 \%$ & $66(69,5 \%)$ & $7(16.7 \%)$ & \\
\hline NA & $9(9.5 \%)$ & $22(52.4 \%)$ & \\
\hline HER2 & & & $<0.0001$ \\
\hline
\end{tabular}




\begin{tabular}{|c|c|c|c|}
\hline Positive & 0 & $10(26.1 \%)$ & \\
\hline Negative & $91(95.8 \%)$ & $26(59.5 \%)$ & \\
\hline NA & $4(4.2 \%)$ & $6(14.3 \%)$ & \\
\hline Site of metastases & & & $0.003^{2}$ \\
\hline Bone & $38(40 \%)$ & $20(47.6 \%)$ & \\
\hline Visceral & $2(2.1 \%)$ & $7(16.7 \%)$ & \\
\hline Soft tissue & $17(17.8 \%)$ & $1(2.3 \%)$ & \\
\hline Bone and visceral & $2(2.1 \%)$ & $10(23.8 \%)$ & \\
\hline Bone and soft tissue & $22(23.2 \%)$ & $2(4.8 \%)$ & \\
\hline Visceral and soft tissue & $7(7.4 \%)$ & 0 & \\
\hline Bone, visceral and soft tissue & $7(7.4 \%)$ & $2(4.8 \%)$ & \\
\hline Number of lesions & & & 0.271 \\
\hline$>3$ & $67(70.5 \%)$ & $27(64.3 \%)$ & \\
\hline$\leq 3$ & $24(25.3 \%)$ & $15(35.7 \%)$ & \\
\hline NA & $4(4.2 \%)$ & 0 & \\
\hline
\end{tabular}

HT: hormone therapy, HIT: hormone-immunotherapy, NA: not available, ${ }^{1} \mathrm{ER}+\mathrm{vs}$ ER-, ${ }^{2}$ visceral vs non visceral

Table 2. First-line salvage HT and additional treatments in the 137 endocrine dependent metastatic breast cancer patients. 


\begin{tabular}{|c|c|c|}
\hline Therapy & Controls (HT) & Cases (HIT) \\
\hline & $\mathrm{N}=95$ & $\mathrm{~N}=42$ \\
\hline \multicolumn{3}{|l|}{ First line hormone-therapy } \\
\hline SERM/SERD, total ${ }^{1}$ & $17(17.9 \%)^{1}$ & $39(92.9 \%)^{1}$ \\
\hline Tamoxifen & 5 & 27 \\
\hline Toremifene & 0 & 12 \\
\hline Fulvestrant & 12 & 0 \\
\hline Al, total & $78(82.1 \%)^{1}$ & $3(7.1 \%)^{1}$ \\
\hline Anastrozole & 8 & 2 \\
\hline Letrozole & 53 & 1 \\
\hline Exemestane & 17 & 0 \\
\hline \multicolumn{3}{|l|}{ Additional treatments } \\
\hline Molecular target therapies ${ }^{2}$ & $28(28.9 \%)^{2}$ & $0(0 \%)^{2}$ \\
\hline Al plus mTOR inhibitors & 8 & 0 \\
\hline Al plus bevacizumab & 6 & 0 \\
\hline Al plus palbociclib & 6 & 0 \\
\hline SERD plus bevacizumab & 1 & 0 \\
\hline SERM plus bevacizumab & 3 & 0 \\
\hline SERD plus palbociclib & 4 & 0 \\
\hline Immunotherapy ${ }^{3}$ & 0 & 42 \\
\hline
\end{tabular}

HT: hormone therapy, HIT: hormone immunotherapy, SERM: selective estrogen receptor modulator, SERD: selective estrogen receptor down-regulator, Al: aromatase inhibitor ${ }^{1} p<0.0001,{ }^{2} p=0.0002,{ }^{3}$ sequential lowdose beta-interferon-interleukin-2 cycles (see materials and methods), among controls, 20 peripremenopausal patients received LHRH agonist for two years at least.

Table 3. PFS and OS unadjusted and adjusted for disease-free interval (DFI), hormone receptor and HER2 status, visceral involvement, Als and biological therapy in patients treated with hormone-immunotherapy (HIT: 0) compared to hormone therapy alone (HT: 1). 


\begin{tabular}{|c|c|c|c|c|c|}
\hline \multirow[t]{2}{*}{ Endpoint } & \multicolumn{2}{|c|}{ Unadjusted analysis } & \multicolumn{3}{|c|}{ Adjusted analysis } \\
\hline & $\mathrm{HR}(95 \% \mathrm{Cl})$ & p-value & $\mathrm{RC}$ & $\mathrm{HR}(95 \% \mathrm{Cl})$ & p-value \\
\hline \multirow[t]{2}{*}{ PFS } & 1.902 & 0.002 & 0.929 & 2.533 & 0.004 \\
\hline & $(1.275-2.837)$ & & & $(1.534-4.738)$ & \\
\hline \multirow[t]{2}{*}{ os } & 1.684 & 0.019 & 0.769 & 2.158 & 0.044 \\
\hline & $(1.089-2.606)$ & & & $(1.021-4.563)$ & \\
\hline
\end{tabular}

PFS: progression free survival, OS: overall survival, RC: regression coefficient. Als: aromatase inhibitors Figures 

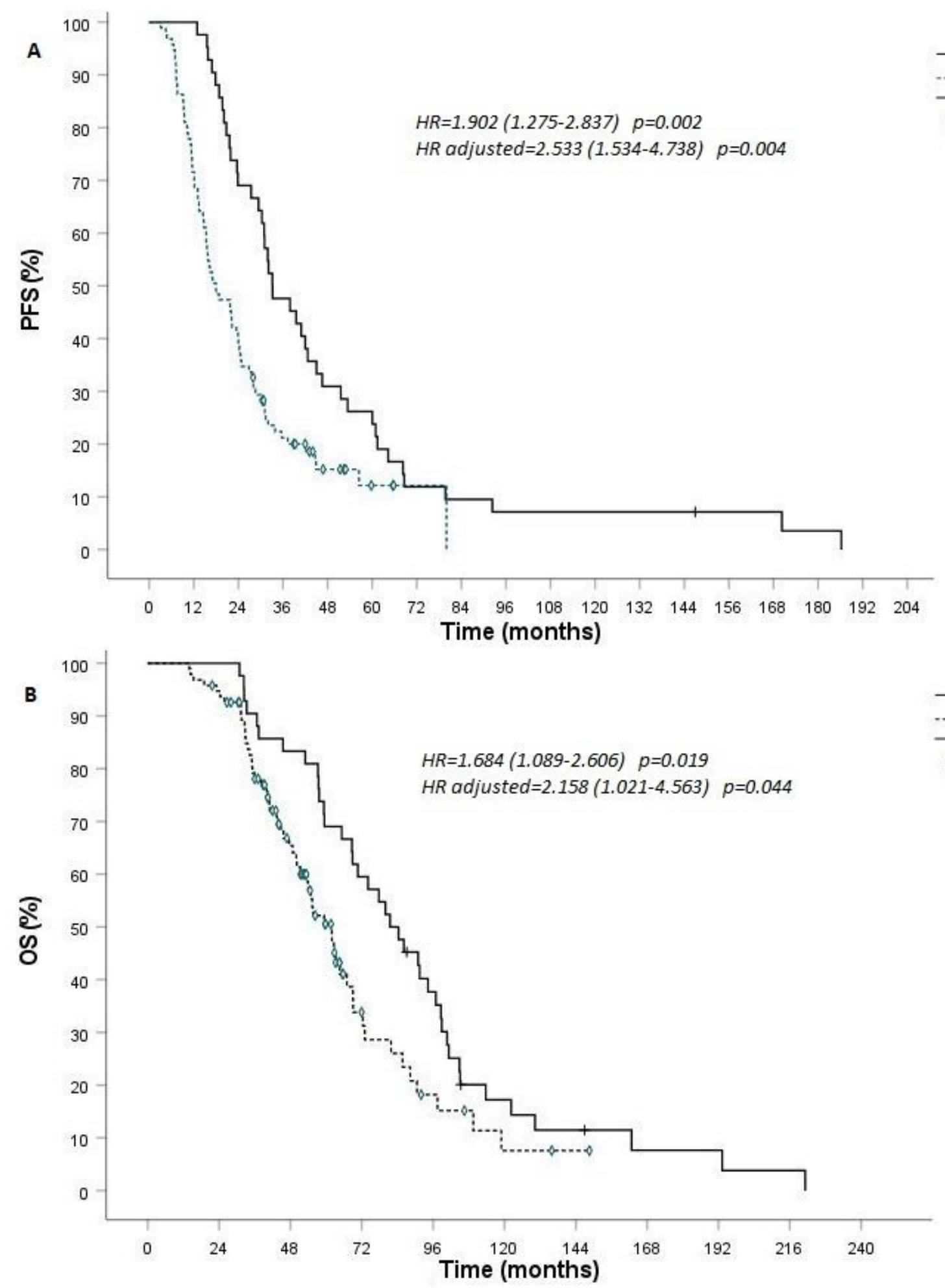

Figure 1

Progression-free survival correlated to hormonal therapy [survival median time of HIT 33 (95\% Cl 24-42), survival median time of $\mathrm{HT} 18$ (95\% Cl 12-23)] (A), Overall survival correlated to hormonal therapy [survival median time of HIT 81 (95\% Cl 64-99), survival median time of HT 62 (54-70)] (B) 